\title{
UNE LIGUE DES CHAMPIONS AVANT L'HEURE ? L'UEFA FACE AU PROJET DE LA COUPE TÉLÉ-MAGAZINE
}

Philippe Vonnard

\section{L'Harmattan | « Sciences sociales et sport »}

2019/1 N 13 | pages 113 à 136

ISSN 1967-7359

ISBN 9782343164007

Article disponible en ligne à l'adresse :

https://www.cairn.info/revue-sciences-sociales-et-sport-2019-1-page-113.htm

Distribution électronique Cairn.info pour L'Harmattan.

(C) L'Harmattan. Tous droits réservés pour tous pays.

La reproduction ou représentation de cet article, notamment par photocopie, n'est autorisée que dans les limites des conditions générales d'utilisation du site ou, le cas échéant, des conditions générales de la licence souscrite par votre établissement. Toute autre reproduction ou représentation, en tout ou partie, sous quelque forme et de quelque manière que ce soit, est interdite sauf accord préalable et écrit de l'éditeur, en dehors des cas prévus par la législation en vigueur en France. Il est précisé que son stockage dans une base de données est également interdit. 


\section{Une ligue des champions avant l'heure? L'UEFA face au projet de la Coupe Télé-Magazine}

\section{Philippe Vonnard}

En date du 19 mai 1967, Marcel Leclerc, homme de presse français et président de l'Olympique de Marseille (OM), soumet au président de l'Union des associations européennes de football (UEFA), le Suisse Gustav Wiederkehr, un règlement d'« un pré-championnat d'Europe latine ${ }^{1} »$. Quel est donc ce projet auquel Marcel Leclerc fait référence ? Et pourquoi cette idée arrive-t-elle sur la table du président de l'UEFA, alors que l'organe régulateur du football européen organise déjà deux compétitions continentales très populaires, à savoir la Coupe des clubs champions européens et la Coupe des vainqueurs de coupe européenne?

Le projet proposé par Marcel Leclerc s'inscrit dans un « air du temps » car, depuis le début des années 1960, l'UEFA - créée en 1954 - doit faire face à d'innombrables demandes de la part d'organismes privés (journalistes, clubs, firmes) qui souhaitent, soit modifier les épreuves européennes déjà en

\section{Philippe Vonnard}

Docteur ès sciences du sport et de l'éducation physique (avec une spécialisation en histoire contemporaine) de l'université de Lausanne philippe.vonnard@unil.ch
Les recherches de Pbilippe Vonnard portent sur
l'bistoire de l'internationalisation des sports. Il
a publie plusieurs articles scientifiques sur cette
thématique et récemment coordonné Building
Europe with the Ball (Peter Lang, 2016),
Beyond Boycotts. Sport during the Cold
War in Europe (De Gruyter, 2017) et écrit
L'Europe dans le monde du football. Genèse
et formation de l'UEFA (Peter Lang, 2018). Il est actuellement chercheur FNS senior à l'Institut des sciences du sport de l'université de Lausanne (ISSUL) et un membre actif du Réseau d'études des relations internationales sportives (RERIS).

\footnotetext{
${ }^{1}$ Lettre de M. LeCLerc à G. Wiederkehr, 19 mai 1967. Archives de l'Union des associations européennes de football (UEFA), cote RM0002713 (Correspondence related to UEFA endorsed competitions), pochette : Coupe télémagazine. Cette recherche a été conduite dans le cadre d'une bourse postdoctorale financée par le Fonds national suisse de la recherche scientifique (FNS). L'auteur souhaite remercier Jean-François Loudcher et les experts pour leurs précieux commentaires. Sa gratitude va également à Léonard Laborie avec lequel il a entretenu des échanges soutenus sur la dimension européenne du football ainsi qu'à Lucile Tonnerre pour sa relecture.
} 
place, soit créer de nouveaux tournois à l'échelle supranationale. Il faut rappeler ici un point important qui peut expliquer cette situation : la première compétition européenne ne provient pas de l'initiative de l'Union, mais des journalistes du quotidien parisien L'Équipe et ce n'est que dans un deuxième temps que l'UEFA s'est emparée de l'organisation de l'épreuve?

La focalisation sur le projet du président de l'OM est intéressante pour au moins trois raisons. Premièrement, celui-ci cristallise les griefs nourris par différents acteurs du football européen à l'encontre du déroulement des compétitions administrées par l'UEFA (nombre de participants, mode d'organisation ou encore discussions sur le calendrier). Deuxièmement, il est développé par un acteur, Marcel Leclerc, qui, en raison de ses activités professionnelles, est capable de soutenir financièrement et techniquement le projet. De plus, en tant que président de l'OM depuis 1965, il côtoie de près d'autres dirigeants du football, qu'ils soient de clubs ou d'instances fédérales. Ainsi, cette position à la fois à l'extérieur et à l'intérieur de « l'espace footballistique européen ${ }^{4}$ » pourrait lui permettre de convaincre les instances européennes du bien-fondé de son idée. Troisièmement, la focalisation sur ce tournoi met au jour un objet qui n'a, jusqu'ici, jamais été traité par l'historiographie des compétitions européennes ${ }^{5}$, à savoir celui des nombreux projets de tournois européens avortés, l’idée de l'homme de presse français ne voyant pas le jour.

\footnotetext{
${ }^{2}$ Montérémal, Gilles. 2007. «L'Équipe : médiateur et producteur de spectacle sportif (19461967) », Le Temps des médias, nº 9, numéro spécial, janvier, p. 107-120.

${ }^{3}$ Sur la reprise de la compétition par l'UEFA voir: VONNARD, Philippe. 2012. La Genèse de la Coupe des clubs champions européens. Une histoire du football européen, coll. «Réflexions sportives », Neuchâtel, CIES. ${ }^{4}$ Gasparini, William ; Polo, Jean-François. 2012. «L'espace européen du football. Dynamiques institutionnelles et constructions sociales », Politique européenne, $\mathrm{n}^{\circ}$ 36, numéro spécial, juin, p. 8-21.

${ }^{5}$ Sans être exhaustif, nous pouvons citer : MitTAG, Jürgen. 2007. «Europa und der Fussball. Die Europäische Dimension des Vereinsfussballs vom Mitropa-Cup bis zur Champions League », in Mittag, Jürgen ; Nieland, Jörg-Uwe (dir.). Das Spiel mit dem Fussball. Interessen, Projektionen und Vereinnabumungen, Essen, Klartext, coll. «Fussball \& Sport», p. 155-176; MourLanE, Stéphane. 2010. «La Coupe latine : aspects sportifs, culturels et politiques (1949-1957)», in REY, Didier (dir.). Football en Méditerranée occidentale de 1900 à 1975, Ajaccio, Éd. Alain Piazzola, p. 53-66 ; Quin, Grégory. 2013. «La Coupe de l'Europe centrale (1927-1938), une compétition internationale oubliée », Stadion. Revue internationale d'bistoire du sport, $\mathrm{n}^{\circ}$ 37, juin, p. 285-304 ; VONNARD, Philippe. 2014. "A Competition that Shook European Football: the Origins of the European Champion Clubs' Cup, 1954-1955 », Sport in History, n 34, décembre, p. 595-619 ; Breull, Xavier ; Constantin Pompiliu-Nicolae. 2015. "The Balkan Cups as a Vector of European Integration », Sport History, n 35, numéro spécial, printemps, p. 591-603; MARSTON, Kevin Tallec. 2016. " "Sincere camaraderie": professionalization, politics and the pursuit of the European idea at the International Youth Tournament, 1948-57 », in VONNARD, Philippe; Quin, Grégory; BanCEL, Nicolas (dir.). Building Europe with the Ball. Turning Points in the Europeanization of Football (19141989), Oxford, Peter LaNG, coll. « Sport, culture and society », p. 137-161.
} 
Le but de cet article consiste principalement à s'interroger sur les causes de cette non-réalisation. Il s'agit notamment de replacer le projet porté par Marcel Leclerc dans le contexte de monopolisation du pouvoir en matière d'organisation de compétitions européennes que s'est réservée, au fil des années, l'UEFA. L'hypothèse émise est que la posture globalement défensive des dirigeants de l'Union vis-à-vis de l'idée du dirigeant marseillais témoigne de leur ambition à renforcer le monopole de l'Union en matière d'organisation de compétitions européennes, et ce afin de consolider la position de l'UEFA en tant qu'organe régulateur du football continental.

L'étude se base sur le dépouillement de documents issus des riches archives de l'UEFA - encore très peu exploitées - en particulier les boîtes relatives aux compétitions européennes. Les procès-verbaux du Bureau fédéral de la Fédération française de football, organisation du pays dont provient le promoteur de l'idée et avec laquelle il entretient des échanges, ont aussi été consultés ${ }^{6}$. En outre, la recherche bénéficie d'une lecture générale de la presse sportive spécialisée française (L'Équipe, France football) des années 1950 et 1960, ces titres abordant très souvent le cas des compétitions européennes dans leurs colonnes, ainsi que de Télé-Magazine, le journal dont Leclerc est le directeur depuis 1955. Enfin, deux entretiens ont été conduits avec Hans Bangerter, secrétaire général de l'UEFA de 1960 à 1989, puis avec son successeur, Gerhard Aigner, qui entre au secrétariat de l'Union en 1969.

L'article est découpé en trois parties. Tout d'abord, nous aborderons le contexte footballistique européen dans lequel le projet s'intègre, et montrerons que les années 1966-1967 apparaissent comme propices à l'élaboration de nouvelles idées en matière de compétitions continentales. Puis, nous détaillerons le projet porté par Marcel Leclerc. Enfin, nous tenterons de comprendre le positionnement de l'instance européenne et les raisons du nonsoutien au projet porté par le président de l'Olympique de Marseille.

\section{I - UNE MULTITUDE DE PROJETS DE COMPÉTITIONS EUROPÉENNES DE CLUBS}

Au moment où Marcel Leclerc lance son projet de compétition européenne, à savoir au printemps 1967, plusieurs compétitions continentales de clubs existent déjà, dont deux sont administrées par l'UEFA.

${ }^{6}$ Il est désormais possible de les consulter en ligne sur la médiathèque de la FFF. 


\section{a) État des lieux d'un calendrier déjà bien chargé}

La première édition de la Coupe des clubs champions européens est organisée lors de la saison 1955-19567. Elle se dispute de septembre à mai en match à aller-retour à élimination directe, et regroupe tous les champions des pays qui composent le territoire de l'Union'. Depuis la saison 1961-1962, et sur le même mode d'organisation, l'UEFA gère également la Coupe des vainqueurs de coupe qui concerne les vainqueurs des coupes nationales? Les deux épreuves sont administrées par deux commissions permanentes distinctes qui ont comme tâche principale de gérer l'organisation de ces compétitions. Ces deux tournois sont très populaires - la Coupe des clubs champions étant toutefois considérée par les suiveurs du jeu comme plus prestigieuse que sa " petite sœur » - et drainent, chaque année, des milliers de spectateurs dans les stades. Ainsi, un article de France football publié à la fin mai 1967 indique que le total de spectateurs de la Coupe des champions est passé de près de 910000 spectateurs pour sa première édition (1955-1956) à près de 2200000 lors de la saison 1964-1965 ${ }^{10}$. Dans son rapport du secrétariat pour les années 1966 et 1967, le secrétaire général de l’UEFA, Hans Bangerter, est plus précis. Il indique que la Coupe des clubs champions a convié en moyenne 27972 spectateurs lors des 65 rencontres que compte l'épreuve, contre 20186 pour les 61 parties de la Coupe des vainqueurs de coupe $^{11}$. Ces chiffres sont considérables, car il faut tenir compte du fait que des petites équipes (comme celles provenant des associations du Danemark, de la Finlande, du Luxembourg ou encore de Malte) n'ayant qu'un faible public sont engagées dans ces deux compétitions. S'il est difficile d'établir une comparaison avec l'assistance lors des matchs des grands championnats européens (comme l'Allemagne, l'Angleterre ou l'Italie), indiquons néanmoins qu'à partir des quarts de finale, les matchs de la Coupe des clubs champions

\footnotetext{
${ }^{7}$ Pour un propos sur le développement de l'épreuve: Vonnard, Philippe. 2016. « How did UEFA Govern the European Turning Point in Football? UEFA, the European Champion Clubs' Cup and the Inter-Cities Fairs Cup Projects (1954-1959)», in Vonnard, P. ; Quin, G. ; BANCEL, N., op. cit., p. 165-185.

${ }^{8} \mathrm{Il}$ peut cependant arriver, occasionnellement, que des associations ne participent pas à l'épreuve. C'est notamment le cas de l'Albanie durant une grande partie des années 1960.

${ }^{9}$ Il n'existe, à ce jour, aucune étude scientifique sur cette compétition. Les propos les plus détaillés sur la création du tournoi restent ceux du journaliste Jacques FERran : Ferran, Jacques. 1978. Les coupes d'Europe, Genève, Famot.

10 «Confidentiel », France football, 30 mai 1967.

11 « Rapport du secrétariat général pour les années 1966 et 1967 », BANGERTER, Hans, janvier 1968. UEFA, cote RM00010068 (Records from former UEFA Library : reports of the General Secretary).
} 
font bien souvent le plein de spectateurs. Mais l'UEFA n'a pas le monopole de l'organisation des épreuves européennes. En effet, à côté de ces deux tournois, d'autres compétitions existent sur le continent.

\section{Tableau. Principales compétitions suprarégionales lors de la saison 1966-1967.}

\begin{tabular}{lccccc}
\hline Nom & Organisateur & $\begin{array}{c}\text { Nb de } \\
\text { clubs }\end{array}$ & $\begin{array}{c}\text { No de } \\
\text { matchs }\end{array}$ & $\begin{array}{c}\text { Nb de } \\
\text { spectateurs }\end{array}$ & $\begin{array}{c}\text { Moyenne de } \\
\text { spectateurs } \\
\text { par match }\end{array}$ \\
\hline $\begin{array}{l}\text { Coupe des clubs } \\
\text { champions européens }\end{array}$ & UEFA & 32 & 65 & 1818228 & 27972 \\
\hline $\begin{array}{l}\text { Coupe des vainqueurs } \\
\text { de coupe européenne }\end{array}$ & UEFA & 32 & 61 & 1231375 & 20186 \\
\hline $\begin{array}{l}\text { Coupe des villes } \\
\text { de foires }\end{array}$ & $\begin{array}{l}\text { Comité privé } \\
\text { basé à Bâle }\end{array}$ & 48 & 94 & 1486500 & 15820 \\
\hline $\begin{array}{l}\text { Coupe internationale } \\
\text { Organisme de } \\
\text { paris sportifs }\end{array}$ & 48 & 144 & 578965 & 4021 \\
\hline
\end{tabular}

Légende : Tableau construit à partir d'informations contenues dans le rapport du secrétaire de l’UEFA pour la saison 1966-1967.

Parmi celles-ci, il faut tout d'abord indiquer la Coupe des villes de foires (Fairs cup). L'épreuve est organisée depuis l'année 1955 par un comité privé basé à Bâle, qui est dirigé par le président de la Fédération internationale de football association (FIFA), sir Stanley Rous. Un peu moins populaire que la Coupe des clubs champions, la Coupe des villes de foires n'en demeure pas moins la plus importante compétition européenne en termes de nombre de participants, puisqu'elle convoque plus de 50 clubs qui s'affrontent en matchs aller et retour à élimination directe de septembre à mai ${ }^{12}$. Le calendrier de l'épreuve se rapproche globalement de celui des tournois administrés par l'UEFA, et il n'est pas rare que les journalistes placent ces épreuves dans les mêmes rubriques. Depuis 1960, plusieurs équipes prennent également part durant la période estivale à la Coupe internationale (nommée également Coupe Intertoto ou Coupe Rappan, voire Championnat d'été), créée par plusieurs organismes de paris sportifs européens. Cependant, son succès reste limité, la moyenne des spectateurs ne dépassant rarement, si nous suivons les

\footnotetext{
${ }^{12}$ Même constat qu'au sujet de la Coupe des vainqueurs de coupe. Pour quelques informations sur la création de l'épreuve, se rapporter à : FERRAN, J., op. cit.
} 
informations données dans les rapports annuels du secrétariat de l'UEFA, 4000 personnes par match.

Parallèlement, des tournois confinés régionalement se disputent tout au long de la saison footballistique. Nous pouvons mentionner ici la Mittel-Europa (Mitropa) cup, qui existait déjà dans l'entre-deux-guerres ${ }^{13}$ et qui a été relancée en 1955. Celle-ci est principalement disputée par des formations autrichiennes, italiennes, hongroises et tchécoslovaques. De même, depuis le début des années 1960, la Coupe des Balkans des clubs réunit des formations de la région. Si ces joutes conservent un fort ancrage régional, il ne faut pas minimiser leur succès. Ainsi, Xavier Breuil et Pompiliu-Nicolae Constantin n'hésitent pas à souligner que la Coupe des Balkans est la compétition la plus populaire de la région après la Coupe des champions ${ }^{14}$.

Dès lors, et sans compter les nombreux matchs amicaux disputés par les clubs tout au long de l'année, constatons que le football européen est le théâtre de très nombreuses confrontations à l'échelle supranationale. Dans son rapport du secrétariat pour l'année 1966-1967, Hans Bangerter peut écrire que, sur le territoire européen, se dispute « dans une période de 12 mois, dans le cadre [des] compétitions, un total de 450 rencontres, ce qui - en tenant compte de l'intersaison d'été et de la pause d'hiver - donne une moyenne hebdomadaire de plus de 10 matchs internationaux par $\operatorname{clubs}^{15} »$. La prédominance des tournois de l'UEFA et le nombre déjà important de compétitions supranationales existantes ne dissuadent pas l'élaboration de nouvelles idées, plus ou moins ambitieuses ${ }^{16}$.

\section{b) Organiser des compétitions de clubs : un enjeu crucial pour l'UEFA}

Ces projets pourraient venir concurrencer les tournois administrés par l'UEFA, situation qui risquerait d'être dommageable pour l'Union, et ce pour trois raisons principales.

Premièrement, au milieu des années 1960, l'UEFA cherche encore à affirmer sa légitimité au sein de l'espace footballistique européen. Gerhard

\footnotetext{
${ }^{13}$ Elle est arrêtée à la fin des années 1930 en raison de la guerre. Sur cette première période de la Mitropa cup, voir : Quin, Grégory. 2016. «Central Europe rules European Football. The "Golden Age" of Regional Connections in European Football (1926-38) », in VonNARD, P. ; Quin, G. ; BANCEL, N., op. cit., p. 137-161.

${ }^{14}$ Breuil, X. ; Constantin, P.-N., op. cit., p. 600.

15 « Rapport du secrétariat général pour les années 1966 et 1967 », Bangerter Hans, janvier 1968. UEFA, cote RM00010068 (Records from former UEFA Library: reports of the General Secretary).

${ }^{16}$ Comme en témoigne la riche correspondance existante dans les boîtes intitulées « endorsed competitions » [compétitions sanctionnées], conservées dans les archives de l’UEFA.
} 
Aigner, qui entre en tant que collaborateur au secrétariat de l'Union en 1969 il en deviendra le secrétaire général en 1989 -, souligne que, durant les années 1960, l'UEFA n'était pas très connue du grand public et plusieurs de ses amis la confondaient avec la FIFA ${ }^{17}$. Mais ce manque de légitimité peut également venir de l'intérieur de l'espace footballistique européen, des acteurs n’hésitant pas à remettre occasionnellement la position de l'Union en question, comme ce fut le cas en 1959 lors de la constitution du Comité interliaison des ligues professionnelles ${ }^{18}$. Sous cet angle, réguler les principales compétitions européennes s'avère crucial pour les dirigeants de l'UEFA dans leur optique d'affirmer davantage l'autorité de l'organisation sur le football européen.

Deuxièmement, d'un point de vue financier, il est très important pour l'UEFA de conserver le succès de ses deux compétitions pour les clubs. En effet, la Coupe des clubs champions et la Coupe des vainqueurs de coupe lui apportent des ressources financières qui lui permettent de soutenir ses activités, mais aussi de les étendre. Outre un droit d'inscription à l'épreuve versé par les participants à l'UEFA - une somme qui en raison de son montant de 200 francs suisses est à considérer comme symbolique -, les clubs qui jouent à domicile doivent donner « un montant équivalant à $3 \%$ de la recette brute ${ }^{19}$ » (avec un minimum de 300 francs suisses) dans un délai de deux mois à l'Union. De plus, elle touche $10 \%$ de la recette brute de la finale (souvent disputée dans un grand stade européen). À la suite des premières négociations avec l'Union européenne de radio (UER) - qui souhaite retransmettre les rencontres de la Coupe des clubs champions de manière régulière sur le réseau Eurovision ${ }^{20}$ qu'elle administre - un premier contrat UER-UEFA est signé en 1961 et voit l'UEFA

\footnotetext{
${ }^{17}$ Entretien avec Gerhard Aigner réalisé le 20 août 2012 à Morges (Suisse).

${ }^{18}$ Organisme créé en mai 1959 par les ligues professionnelles d'Angleterre, de France et d'Italie et qui a notamment pour but de créer des compétitions supranationales (comme la Coupe des Alpes lancée en 1960). Après différentes tractations, en 1963, le Comité des ligues est finalement englobé dans une commission permanente de l'UEFA intitulée «Commission du football professionnel et non amateur ».

${ }^{19}$ " Coupe des clubs champions européens. Règlement. Saison 1966/1967 [article 17, al. 3] », WiEDERKEHR, Gustav ; BANGERTER,Hans, mai 1966. Document que nous a gentiment transmis le documentaliste de l'UEFA. La même règle s'applique aussi à la Coupe des vainqueurs de coupe.

${ }^{20}$ L'Eurovision a été mis en place en 1954. Si nous suivons le descriptif donné par Patrick AlvÈs, en 1967, le réseau touche presque une vingtaine de pays et comprend « $102000 \mathrm{~km}$ de circuits, c'est-à-dire $90000 \mathrm{~km}$ de relais hertziens et $12000 \mathrm{~km}$ de câbles, et $2500 \mathrm{sta}$ tions d'émission, qui desservent plus de 55 millions de récepteurs de télévision représentant 250 millions de téléspectateurs ». Alvès, Patrick. 2008. "L'Union européenne de radiodiffusion (1950-1969) ", in LÉvy, Marie-France ; SicARD, Marie-Noëlle (dir.), Les lucarnes de l'Europe. Télévisions, cultures, identités, 1945-2000, Paris, Publications de la Sorbonne, coll. «Internationale », p. 56.
} 
recevoir une somme fixe pour la retransmission des finales ${ }^{21}$. Concernant les matchs des autres tours, s’il est décidé que la négociation des indemnités de la télévision est l'apanage des clubs - moyennant l'accord avec la fédération nationale à laquelle ils sont affiliés -, $25 \%$ du montant de la somme doit être transféré à l'UEFA. Ces montants, qui correspondent à environ trois quarts des recettes de l'Union ${ }^{22}$, permettent de soutenir la politique ambitieuse que mène le nouveau président de l'organisation, Gustav Wiederkehr. En effet, dès sa nomination en 1962, il s'attelle à étendre les prérogatives de l'UEFA. Ainsi, au moment où Marcel Leclerc développe son projet, l'Union compte 15 commissions permanentes qui traitent de sujets spécifiques (arbitrages, compétitions, aspects techniques du jeu, mais aussi des aspects juridiques) contre 4 au début des années 1960. Cet accroissement des tâches a pour conséquence une augmentation des frais d'administration (en raison de l'engagement de nouveaux collaborateurs et des frais relatifs aux réunions des commissions, l'UEFA prenant à sa charge le séjour et le déplacement des membres). De même, l'Union œuvre dorénavant au développement du football européen, organise différents cours (pour arbitres et entraîneurs) et dispose également de fonds spéciaux (alimentés directement par les sommes perçues de la télévision) destinés par exemple à financer le déplacement des équipes juniors provenant des petites associations nationales pour son tournoi international annuel. Il est donc très important que l'UEFA sécurise le succès de ses compétitions, et c'est pourquoi son élite dirigeante s'inquiète d'un infléchissement de la moyenne des spectateurs qui se repère depuis le début de la décennie, autant dans la Coupe des clubs champions que dans la Coupe des vainqueurs de coupe.

Troisièmement, ces compétitions jouent un rôle très important en tant que ciment entre les différentes associations nationales de l'organisation $^{23}$. Or, à partir du début des années 1960 et la création de l'Entente de

\footnotetext{
${ }^{21}$ Pour un aperçu général des rapports entre les dirigeants de l'UEFA et ceux de l'UER : MitTAG, Jürgen ; Nieland, Jörg-Uwe. 2013. " Auf der Suche nach Gesamteuropa: UEFA und EBU als Impulsgeber der Europäisierung des Sports ", in BerTling, Christoph ; Mertin, Evelyn (dir.). Freunde oder Feinde? Sportberichterstattung in Ost und West während des Kalten Kriegs, Gütersloh, Medienfabrik Gütersloh, p. 208-229. Et pour une discussion sur ces premières négociations : VONNARD, Philippe; LABORIE, Léonard, sous presse. «L'invention d'un rendez-vous médiatique européen. L'UEFA, l'UER et la Coupe des clubs champions européens de football (1956-1968)», Vingtième siècle. Revue d'histoire.

${ }^{22}$ Chiffre à prendre comme une estimation. Celle-ci devra être vérifiée dans de futurs travaux.

${ }^{23}$ Pour une réflexion préliminaire sur cet aspect: Vonnard, Philippe ; Marston, Kevin Tallec. 2017. «Building bridges between separated Europeans: the role of UEFA's competitions in East-West exchanges (1955-1964)», in Vonnard, Philippe ; SBetTi, Nicolas; Quin, Grégory (dir.). Beyond boycotts. Sport during the cold war in Europe, De GruYTER, Oldenburg, 2017, coll. « Rethinking the Cold War », p. 85-108.
} 
Florence, il est acquis que trois blocs existent au sein de l'UEFA : le bloc des pays communistes, le bloc des pays anglo-scandinaves et l'Entente de Florence (formée des pays latins et du Benelux). Cette situation n'est pas sans créer des tensions entre les associations nationales et s'ajoute au contexte de la Guerre froide. Car il faut rappeler ici un fait important, à savoir que l'UEFA est composée de pays politiquement divisés ${ }^{24}$. En effet, depuis sa création, elle voit la cohabitation des pays du bloc de l'Est et de l'Ouest, surpassant ainsi les rivalités dues à la Guerre froide ${ }^{25}$. Même si l'historiographie récente de ce conflit tend désormais à souligner que les deux blocs étaient, certes, divisés mais pas totalement déconnectés ${ }^{26}$, soulignons que très rares sont les autres organisations se réclamant « européennes 》- même sportives ${ }^{27}$ - créées après la Deuxième Guerre mondiale qui bénéficient d'un tel agencement. Dès lors, si les dirigeants s'emploient à minimiser l'influence de ce contexte au sein de l'Union ${ }^{28}$, la Guerre froide influe, en certaines circonstances ${ }^{29}$, sur les relations entre les associations membres de l'Union. Hans Bangerter, qui devient secrétaire général de l'UEFA en 1959, nous a d'ailleurs indiqué que la politique était l'un des principaux problèmes auxquels il devait faire face

\footnotetext{
${ }^{24}$ Sur la création de l'UEFA : VonnARD, Philippe. 2018. L'Europe dans le monde du football. Genèse et formation de l'UEFA, coll. « Euroclio », Bruxelles, P.I.E. Peter Lang.

${ }^{25}$ Sur ce point voir : MitTaG, Jürgen. 2014. « Negotiating the Cold War? Perspectives in Memory Research on the UEFA, the Early European Football Competitions and the European Nations Cups », in Pyta, Wolfram; Havemann, Nils (dir.). European Football and Collective Memory, Palgrave Macmillan, Basingstoke, coll. «Football in an Enlarged Europe », p. 40-63.

${ }^{26}$ Pour un exemple de ce type de réflexion voir : Hochscherf, Tobias ; Laucht, Christopher ; Plowman, Andrew (dir.). 2010. Divided, but not Disconnected: German Experiences of the Cold War, New York, Berghahn Book.

${ }^{27}$ Très peu d'études existent à ce jour sur les organisations européennes sportives. Nous pouvons citer comme exception : Roger, Anne ; TERret, Thierry. 2012. European Athletics. Une histoire continentale de l'athlétisme, Stuttgart, Neuer Sportverlag. Et de manière plus latérale, voir aussi quelques contributions dans : Archambault, Fabien ; ArTiaga, Loïc ; Bosc, Gérard (dir.). 2015. Le continent basket. L'Europe et le basket-ball au XX' siècle, Bruxelles, PI.E. Peter Lang, coll. «Euroclio ». Et également : Loudcher, Jean-François ; DAY, Dave. 2013. «The International Boxing Union (1913-1946): A European Sports and/or Political Failure? ", The International Journal of the History of Sport, $\mathrm{n}^{\circ}$ 30, novembre, p. 2016-2030.

${ }^{28}$ Mittag, Jürgen, Vonnard, Philippe, 2017. "The role of societal actors in shaping a pan-European consciousness. UEFA and the overcoming of Cold War tensions, 1954$1959 »$, Sport in history, $n^{\circ} 37$, numéro spécial, septembre, p. 332-352.

${ }^{29}$ C'est par exemple le cas lorsque l'Espagne franquiste refuse d'affronter l'Union soviétique lors de la Coupe d'Europe des nations en 1960. De même, dans la première partie de la décennie, l'Allemagne de l'Est a de nombreuses difficultés à obtenir les visas en vue du déplacement de ses équipes nationales (juniors et élites).
} 
durant les années $1960^{30}$. En conséquence, les épreuves continentales permettent, chaque année, de perpétuer les liens entre les associations et il est donc impératif pour ses dirigeants que l'UEFA conserve leur administration.

Ces raisons administratives, économiques et politiques expliquent que les dirigeants du comité exécutif de l'Union cherchent à consolider la prédominance des compétitions UEFA sur les autres tournois existants. Depuis le début des années 1960, ils prennent des mesures en ce sens et confèrent notamment à l'UEFA le droit unique d'organiser des tournois qui se disputent sur tout le Vieux Continent ${ }^{31}$, exception faite de la Coupe des villes de foires. De plus, ils décident que les règlements des compétitions supranationales devront dorénavant être impérativement validés par son comité exécutif. Au milieu de la décennie, cette politique tend à se renforcer. En effet, en 1966, et après des discussions informelles commencées depuis quelques années déjà, le congrès ordinaire de l'UEFA, réuni à Londres, décide que l'entité reprendra dans un futur proche l'organisation de la Coupe des villes de foires ${ }^{32}$. En outre, à la fin de l'année, l'UEFA refuse la mise sur pied d'un projet qui lui est présenté par le Service de presse et d'information de la Communauté économique européenne (CEE) et qui consiste dans l'organisation d'une Coupe du Marché commun. Cette idée est ambitieuse, puisqu’il s'agirait de convoquer les deuxièmes des championnats des six pays formant le Marché commun $^{33}$ auxquels s'additionneraient une formation anglaise et une équipe écossaise $^{34}$. La décision confirme l'ambition des dirigeants de l'Union de se réserver le droit unique de gérer les compétitions continentales et, surtout, de ne pas permettre à des organismes extérieurs au domaine footballistique de créer des tournois supranationaux d'envergure qui pourraient s'avérer une menace pour le succès de la Coupe des clubs champions et de la Coupe des vainqueurs de coupe.

\footnotetext{
${ }^{30}$ Entretien avec Hans BangerTer réalisé le $1^{\text {er }}$ octobre 2012 à Böllingen (Suisse).

31 «Toute compétition ouverte aux clubs de toutes les associations membres ne peut être organisée que par l'UEFA elle-même. » Statuts de l'UEFA, édition 1962 [article 21, alinéa 3], Wiederkehr, Gustav ; Bangerter, Hans, 17 juillet 1962. UEFA, cote RM00005779 (Statutes of UEFA, 1954-1976).

32 « Procès-verbal de la VIII" assemblée générale de l'UEFA du 6 juillet 1966 au "Royal Garden Hotel”, Kensington Gardens, Londres W8», Bangerter, Hans, juillet 1966. UEFA, cote RM00005990 (VIII Ordinary Congress, London). Cependant, signe de la difficulté et des résistances sur le sujet, il faudra attendre 1971 pour que cette reprise soit effective.

${ }^{33}$ Allemagne, Belgique, France, Italie, Luxembourg et Pays-Bas.

${ }^{34}$ Lettre de Jacques-René RABIER à l'UEFA, 18 novembre 1966. UEFA, cote RM0002713 (Correspondence related to UEFA endorsed competitions), pochette : Coupe marché commun.
} 
C'est donc dans ce contexte de renforcement de l'autorité de l'UEFA en matière d'organisation de compétitions continentales que Marcel Leclerc développe son idée de tournoi européen.

\section{II - UNE NOUVELLE COMPÉTITION EUROPÉENNE ? LE PROJET DE COUPE TÉLÉ-MAGAZINE}

Dès les premières lignes du projet de règlement de l'épreuve, concocté en mai 1967, Marcel Leclerc indique :

«Tout le monde s'accorde à reconnaître qu'une compétition régulière, comportant davantage de matchs entre les principaux clubs étrangers que l'actuelle Coupe d'Europe, s'impose. Tant sur le plan sportif et celui de la relance d'intérêt que sur le plan financier ${ }^{35}$.»

Selon cette vision, la "Coupe d'Europe ", c'est-à-dire la Coupe des clubs champions, ne serait plus suffisante. C'est une attaque forte contre l'UEFA, car Marcel Leclerc s'en prend ici à son épreuve reine. Quels sont les griefs du dirigeant marseillais envers cette épreuve et comment souhaite-t-il y répondre?

\section{a) Imposer un nouveau mode d'organisation des compétitions européennes}

Une des critiques majeures concerne l'aspect financier de ce tournoi, qui ne rapporterait pas assez d'argent aux clubs qui y sont engagés. Au contraire, la nouvelle compétition proposée par Leclerc se donne explicitement comme but d'apporter à ses participants « un intéressant supplément de recettes ${ }^{36}$ ». Plusieurs propositions sont faites en ce sens, et notamment la garantie que les clubs joueront un nombre fixe de matchs à domicile. En outre, le projet de règlement prévoit que les formations engagées dans le tournoi toucheront une recette de 80000 francs français sur chaque rencontre jouée à l'extérieur. Afin d'assurer un succès financier aux participants, le promoteur de l'épreuve envisage « de n'admettre dans cette compétition que des clubs présentant, en plus d'une valeur sportive suffisante, des garanties financières et des possibilités de recettes suffisantes ${ }^{37} »$. Le versant commercial du projet contraste

\footnotetext{
${ }^{35}$ « Coupe Télé-Magazine (pré-championnat d'Europe latine) - sur invitation. Projet », LECLERC, Marcel, mai 1967. UEFA, cote RM0002713 (Correspondence related to UEFA endorsed competitions), pochette : Coupe télémagazine.

${ }^{36}$ Ibid.

${ }^{37}$ Ibid.
} 
donc fortement avec les épreuves administrées par l'UEFA, dont le seul critère de participation est d'ordre sportif (remporter les épreuves nationales, championnat ou coupe). De plus, dans le cadre de celles-ci, c'est uniquement le club qui joue à domicile qui empoche la recette du match ${ }^{38}$.

Par ailleurs, Leclerc imagine que les sommes provenant de la billetterie seraient complétées par l'apport des sociétés de télévision qui sont considérées comme étant des actrices essentielles de la future compétition. Promoteur de la " télédiffusion visuelle », il lance l'hebdomadaire Télé-Magazine en 1955, au sein duquel il milite rapidement pour un renforcement des liens entre la télévision et les clubs de football ${ }^{13}$. Fait révélateur de cette posture, il propose dans son projet une répartition précise des recettes et émet l'idée de retransmettre un match à un horaire décalé (la veille de la majorité des rencontres) dans le but d'assurer la publicité de l'épreuve. Certes, plusieurs rencontres des tournois de l'UEFA sont désormais retransmises par le réseau Eurovision. Cependant, jusqu'en demi-finales, c'est au club de négocier directement les droits de retransmission avec les sociétés de télévision. Or, des frictions existent entre les dirigeants de grands clubs et ceux des associations nationales qui restent globalement frileux face à un acteur qu'ils considèrent comme une potentielle menace ${ }^{40}$. Même si depuis le début des années 1960, des avancées sont faites, ce qui permet notamment la retransmission de la plupart des rencontres des équipes nationales et des grands matchs de la Coupe des champions, deux griefs principaux sont toujours nourris par les instances footballistiques à la télévision. D’une part, le risque de perte d'affluence si le match est disputé en même temps que d'autres parties sur le territoire national, l'argument défendu étant que le public préférera regarder le match à la télévision que de se déplacer dans les stades. D'autre part, le risque de baisse du nombre de pratiquants, notamment chez les jeunes qui seraient davantage attirés par les rencontres télévisées que par la pratique

${ }^{38}$ À noter que le club recevant a l'obligation de couvrir les frais d'organisation du match ainsi que le séjour de l'équipe visiteuse durant trois jours.

39 À plusieurs occasions, entre 1955 et 1964 - année pendant laquelle un accord est trouvé entre les dirigeants de la Fédération française et ceux de la télévision - le magazine propose au lecteur des débats sur la question. Par exemple, Leclerc écrit en décembre 1955 un éditorial intitulé : «Football-Télévision : un dialogue de sourds-muets!», LECLERC, Marcel, Télévision programme Magazine, $\mathrm{n}^{\circ}$ 8, 18 au 24 décembre 1955. Après l'accord de 1964, il est mentionné dans la page des programmes avant le match France-Hongrie : «Ainsi, huit ans après le rugby, le football se met à l'heure de la télévision », Télé-Magaz̧ine, n 444, 25 avril au $1^{\text {er }}$ mai 1964.

${ }^{40}$ Meyer, Jean-Christophe. 2015. « L'essor du football télévisé à l'ère du monopole public (1950-1966) : un vecteur d'identité nationale et européenne ", in ZoudjI, Bachir ; REY, Didier (dir.). Le football dans tous ses états. Évolutions et questions d'actualité, Bruxelles, De Boeck, p. $279-288$. 
elle-même. Cette posture a comme conséquence que, selon des promoteurs des médias comme Leclerc, le potentiel déjà existant de la télévision n’est pas totalement exploité, ce qui est dommageable autant pour les téléspectateurs qui ne voient pas assez de football à la télévision - que pour les clubs - dont les recettes restent limitées.

Enfin, les modalités de la future compétition choisies par Marcel Leclerc répondent à certaines critiques émises par différents acteurs (dirigeants de clubs, journalistes) sur le système à élimination directe qui prévaut dans les tournois de l'UEFA. Ainsi, un journaliste reconnu comme Jacques Ferran ${ }^{41}$ souligne que ce genre de procédé implique des rencontres entre les favoris à la victoire finale, et ce, dès le premier tour de la compétition. Il regrette que, dans l'édition 1967-1968, Celtic Glasgow rencontre Dynamo Kiev et que Real Madrid soit opposé si tôt dans l'épreuve à Ajax Amsterdam alors que, dans le même temps, le champion du Luxembourg affronte celui d'Islande ${ }^{42}$. Or, Ferran se demande s'il ne serait « pas logique de donner la possibilité aux clubs champions [à comprendre ceux des grands championnats] qui viennent de consentir des efforts considérables pour enlever le titre national, et pour y faire honneur, de disputer plusieurs rencontres avant leur élimination ${ }^{43} »$. Ces différents griefs sont notamment discutés lors d'une table ronde semi-officielle qui se tient à Monaco les 11 et 12 mars 1967. Organisée à l'initiative du prince Rainier - « grand ami du football ${ }^{44} »-$, la manifestation regroupe 25 clubs européens provenant de 11 pays $^{45}$, le vice-président de

\footnotetext{
${ }^{41}$ Jacques Ferran entre à L'Équipe à la fin des années 1940 et y restera jusqu'aux années 1990. En hiver et au printemps 1955, il participe activement au développement du projet de Coupe d'Europe des clubs portés par L'Équipe. Devenu rédacteur en chef de France football, au milieu des années 1960, c'est donc un journaliste respecté et qui n’hésite pas dans la lignée de ses prédécesseurs, Gabriel HANOT et Jacques DE RYSWICK, à utiliser les colonnes de L'Équipe et de France football pour proposer des idées en vue de dynamiser les échanges footballistiques européens.

${ }^{42}$ «L'occasion était belle d'aller plus loin », Ferran, Jacques, France football, 11 juillet 1967.

43 «L'engrenage de Monaco », Ferran, Jacques, France football, 14 mars 1967.

${ }^{44}$ " La table ronde de Monaco se présente bien : même Manchester sera représenté ! ", FERRAn, Jacques, L'Équipe, 9 mars 1967.

${ }^{45}$ Les clubs participants sont (par ordre alphabétique de leur pays) : Manchester United (Angleterre) ; Austria Vienne (Autriche); Anderlecht et Standard de Liège (Belgique); FC Barcelone et Real Madrid (Espagne) ; Girondins de Bordeaux, Olympique de Marseille, AS Monaco, OGC Nice, AS Saint-Étienne et RC Strasbourg (France); Panathinaikos Athènes (Grèce), Juventus de Turin, AC Milan et Naples (Italie) ; Feyenoord Rotterdam (PaysBas) ; Benfica Lisbonne et FC Porto (Portugal) ; Servette de Genève, FC La Chaux-deFonds et Lausanne-sport (Suisse); Dukla Prague (Tchécoslovaquie). « À la table ronde de Monaco. Lois du jeu et compétitions grands sujets de discussion », Ferran, Jacques, L'Équipe, 11 et 12 mars 1967.
} 
l'UEFA, José Crahay - présent à titre personnel -, Jacques Ferran représentant de l'Association internationale de la presse sportive (AIPS) ainsi qu'une vingtaine de journalistes provenant de plusieurs pays d'Europe. Le but du congrès est de discuter de différentes problématiques liées au football et, parmi celles-ci, du futur des compétitions européennes. Si différentes propositions de modifications des règlements des épreuves en place, voire des projets de nouveaux tournois, sont présentés, les participants s'arrêtent finalement sur quelques vœux qu'ils transmettent à l'UEFA. Un de leurs principaux souhaits est de revoir, à terme, le mode d'organisation de la Coupe des champions, en remplaçant les deux premiers tours de la compétition par une phase de groupe. Il s'agirait en particulier de créer quatre poules de quatre équipes, les deux premiers de chaque groupe étant ensuite qualifiés pour les quarts de finale. Ce procédé aurait comme avantage d'augmenter le nombre de rencontres et d'assurer aux équipes participantes de disputer un minimum de six matchs.

Marcel Leclerc est présent à Monaco et a même l'occasion d'exposer son idée. C'est sans doute pour cela que les propositions émises sur le Rocher se retrouvent dans son projet. En effet, la future épreuve comprendrait deux phases distinctes. Pour le premier tour, les 12 équipes convoquées seraient divisées en 2 poules de 6 , et joueraient chacune en match aller et retour contre chaque équipe. Une phase finale verrait ensuite les formations de chaque poule s'affronter en match aller et retour selon leur classement respectif. Dès lors, les clubs participants seraient assurés de jouer au minimum 12 matchs, dont au moins 6 matchs à domicile. En comparaison de la Coupe des clubs champions, les possibilités de gains sont conséquentes, puisqu'un club qui atteint la finale de l'épreuve phare de l'UEFA ne peut, tout au mieux, disputer que quatre matchs à domicile et neuf au total.

Dans un premier temps, Leclerc ne propose pas une compétition européenne mais envisage de convoquer des équipes espagnoles, françaises et italiennes. Ce choix n'est pas anodin pour le succès du tournoi, les footballs espagnol et italien étant alors perçus comme les meilleurs d'Europe du point de vue du niveau de jeu ; mais ceux aussi qui disposent des clubs ayant le plus de capacité financière et les plus grands stades. En outre, le projet du président de l'Olympique de Marseille se place en continuité de la Coupe latine, tournoi disputé de 1949 à 1957 entre des clubs des trois pays concernés et auxquels s'ajoutait le Portugal ${ }^{46}$. Toutefois, comme le note un journaliste de L'Équipe, dans l'esprit de Marcel Leclerc, cette épreuve est l'avant-goût d'un futur championnat d'Europe des clubs ${ }^{47}$. Dans le projet de règlement,

\footnotetext{
${ }^{46}$ Voir Mourlane, S., op. cit.

${ }^{47}$ «Le “Championnat latin” : réunion le 26 juin », L'Équipe, 16 juin 1967.
} 
son promoteur note d'ailleurs que le tournoi devra rapidement s'étendre à d'autres pays, en premier lieu la Belgique ou la Suisse. À cet effet, il a déjà noué des premiers contacts et notamment rencontré, au mois de mai 1967, un autre dirigeant désireux de dynamiser les compétitions européennes de clubs, le président du club bruxellois d'Anderlecht, Albert Roosen ${ }^{48}$.

Le projet présenté par le président de l'OM est donc ambitieux et sous plusieurs aspects comporte des nouveautés par rapport aux compétitions de l'UEFA. À ce stade de l'analyse, deux nouvelles questions se posent : tout d'abord, pourquoi Marcel Leclerc s'engage-t-il dans une telle entreprise ? Puis, comment expliquer son choix de créer une épreuve à l'échelle européenne?

\section{b) Un projet aux multiples enjeux}

Plusieurs facteurs expliquent la démarche du dirigeant marseillais. En premier lieu, il peut s'agir d'une opportunité pour lui de remettre définitivement l'OM sur les rails du succès. En effet, en 1965, Marcel Leclerc a repris les rênes du club alors qu'il était au bord de la faillite et qu'il végétait péniblement en deuxième division ${ }^{49}$. Passionné de sport - il a pratiqué, dès sa jeunesse, la natation (" champion provençal du cent mètres de nage libre au sein du Cercles des nageurs de Marseille $\left.{ }^{50} »\right)$, le volley-ball et le football (joueur de l'équipe amateur de l'OM) -, au début des années 1950, il est notamment responsable de la rubrique volley-ball au Méridional ${ }^{51}$, un journal provençal. Ainsi, pour «le Parisien »- comme le surnomment les supporters de l'OM en raison du fait que ses activités professionnelles se trouvent dans la capitale - reprendre le club olympien c'est autant assouvir sa sincère passion pour le sport que d'objectiver son attachement à une ville où il a vécu de nombreuses années. Après des débuts difficiles qui voient notamment le nouveau président entrer en conflit avec la Mairie - et en particulier avec l'emblématique maire de la ville, Gaston Deferre ${ }^{52}$ - au sujet de l'utilisation du stade Vélodrome ${ }^{53}$, lors de la saison 1966-1967, Marcel Leclerc est en

\footnotetext{
${ }^{48}$ «Le championnat latin que mijote l'OM », France football, 13 juin 1967. En 1966, Albert Roosen soutient en particulier l'idée de Coupe du marché commun citée ultérieurement.

${ }^{49}$ L'année 1965 voit l'OM connaître la pire affluence de son histoire, puisque le match contre Forbach ne réunit que 500 spectateurs.

${ }^{50}$ Boquillon, Laurent. 2010. "L'Olympique de Marseille de Marcel Leclerc : l'accession d'une "victime" au pouvoir (1965-1966)», in Rey, D., op. cit., p. 146.

${ }^{51}$ «La mort de Marcel Leclerc », Peroni, Victor, L’Équipe, 22 avril 1983.

${ }^{52}$ Gaston Deferre est un acteur majeur de la politique hexagonale des années 1950 aux années 1980. Plusieurs fois ministre, il est maire de Marseille, d'abord brièvement de 1945 à 1946, puis occupe le poste durant un très long mandat, à savoir de 1953 à 1986.

${ }^{53}$ Sur ce sujet, voir : Boquillon, L., op. cit.
} 
phase de réussir son pari en stabilisant la formation phocéenne dans l'élite du football français. Homme ambitieux, il souhaite cependant que le club retrouve rapidement les sommets de la hiérarchie sportive ${ }^{54}$. Sous cet angle, le tournoi supranational qu'il propose peut servir cet objectif, car la nouvelle épreuve apporterait assurément une manne financière supplémentaire au club, lui permettant, d'une part, d'être plus conquérant sur le marché des transferts et, d'autre part, d'acquérir une plus grande visibilité en France, mais aussi en Europe, ce qui pourrait inciter les meilleurs joueurs à venir exercer leur art sur la Canebière.

En deuxième lieu, la compétition pourrait aussi bénéficier à ses activités commerciales. Âgé d'une quarantaine d'années, Marcel Leclerc est alors au sommet de sa carrière professionnelle. Après des débuts modestes, Télé Magażine - qui tout au long de ses 80 pages est consacré à tout ce qui touche à la télévision, autant ses programmes que ses animateurs et animatrices vedettes - est devenu un hebdomadaire incontournable de la presse française. Édité à près de 400000 exemplaires ${ }^{55}$, il se situe dans le sillage de ses principaux concurrents (Télé 7 jours du groupe Hachette ou encore Télé-poche), profitant de l'extension croissante de la télévision au sein de la population française ${ }^{56}$. Dès lors, cette nouvelle épreuve constitue un moyen pour Leclerc, dans un marché très concurrentiel, d'étoffer l'actualité de son magazine afin de dynamiser les ventes en misant sur un sujet - le football ${ }^{57}$ - très vendeur; de gagner de nouvelles parts de marché sur le territoire français (les équipes de Bordeaux et de Nantes sont par exemple pressenties pour accompagner Marseille dans la première édition du tournoi), voire de diversifier son offre afin de se démarquer de ses concurrents.

En troisième lieu, il est possible d'imaginer que Leclerc poursuit une ambition publique, voire politique. En effet, être président de l'OM, c'est occuper une place importante dans la société marseillaise. Ainsi, au travers de son action pour le club, Marcel Leclerc peut espérer accéder à un rang élevé

\footnotetext{
${ }^{54}$ L'OM a été une des meilleures équipes des années 1930, le club remportant un championnat et deux fois la Coupe de France.

${ }^{55}$ Pour un aperçu sur les magazines de l'époque voir : DAKHLIA, Jamil. 2001. «Variations sur la télélecture. Les discours de la presse de programmes en France », Réseaux, n 105, hiver, p. 131-159.

${ }^{56}$ Selon Agnès Chauveau et Philippe Tétart, le nombre de postes de télévision en France passe de 4,4 millions en 1964 à 14 millions en 1974. Chauveau, Agnès ; Tétart, Philippe. 1999. Introduction à l'histoire des médias en France. De 1881 à nos jours, Paris, Armand Colin, coll. «Synthèse », p. 67.

${ }^{57}$ En septembre 1969, Télé-Magazine lance un hebdomadaire uniquement consacré au football qui est intitulé But. Télé-magažine, n 725, 13 au 19 septembre 1969.
} 
de notabilité locale ${ }^{58}$. Mais, il semble voir plus loin et espère utiliser le club comme tremplin politique. Dans le courant de l'année 1967, il se présente à des élections dans le $1^{\text {er }}$ arrondissement de Marseille, toutefois sans succès.

Enjeux sportifs, économiques, voire politiques se conjuguent et expliquent l'action du président de l'Olympique de Marseille. À l'aune de ces éléments, l'idée de créer une épreuve européenne prend tout son sens, car si nous savons désormais que le football est un jeu marqué, depuis sa diffusion en Europe à la fin du XIX ${ }^{\mathrm{e}}$ siècle, par de nombreux échanges transnationaux ${ }^{59}$, la volonté de mettre sur pied une compétition européenne ne va néanmoins pas de soi. À ce titre, il n'est pas possible pour Marcel Leclerc d'inventer une nouvelle épreuve nationale, le championnat français étant désormais solidement ancré6 ${ }^{60}$. En revanche, l'échelle continentale se présente toujours comme une alternative possible. En conséquence, le dirigeant marseillais semble utiliser ici le cadre européen comme « un argument ${ }^{61}$ », pour reprendre le terme développé par Matthieu Flonneau au sujet des promoteurs du réseau autoroutier en France.

Marcel Leclerc a plusieurs atouts à faire valoir pour défendre son projet, et en particulier des soutiens dans le monde des médias (presse et télévision). De plus, avec son poste de président de l'Olympique de Marseille, il est un acteur actif de l'espace footballistique - certes, quelque peu atypique, ce qui se verra notamment lors de ses démêlés avec la justice qui font suite à son départ de l'OM en $1972^{62}$ - et nous pouvons supposer (bien que

${ }^{58}$ Sur le football comme vecteur de notabilité locale voir : SснотTÉ, Manuel. 2016. «Monter en première division. Trajectoires de notabilisation des présidents de clubs de football professionnel (1960-1999), Politix, n 114, juin, p. 99-120.

${ }^{59}$ Sur l'importance de ces échanges transnationaux dans le développement du football voir notamment : Lanfranchi, Pierre. «Football, cosmopolitisme et nationalisme », Pouvoirs, $\mathrm{n}^{\circ}$ 101, p. 15-25 ; Koller, Christian. 2009. «Einlatung : Europäischer Fussball im Zeithaler der Katastrophen », in Kolder, Christian ; Brändle, Fabien (dir.). Fussball zwischen den Kriegen, Zurich, Lit Verlag, p. 1-22 ; Dietschy, Paul. 2016. «Football during the Belle-Epoque: The first "Europe du football” (1903-1914)», in Vonnard, P., Quin, G., Bancel, N., op. cit., p. 21-51.

${ }^{60}$ La division 1, comme elle est alors appelée, a été créée en 1932. Sur son développement voir : Ramsay, Arnaud ; Dietschy, Paul. 2013. Ligue 1, 80 ans de football professionnel : le championnat de France depuis 1932-1933, Paris, Solar.

${ }^{61}$ Flonneau, Matthieu. 2017. " "Route en or" et "automobile pour tous”. Adosser l'archéologie des autoroutes françaises à l'horizon européen », in BOUvIER, Yves ; LABOrIE, Léonard (dir.). L'Europe en transitions. Énergie, mobilité, communication XVIII'-XXI siècle, Paris, Nouveau Mondes éditions, p. 192.

${ }^{62}$ Déjà inquiété par la justice pour une affaire de pronostics au début des années 1970, dès septembre 1972, Leclerc est accusé par le comité directeur de l'OM d'avoir détourné de l'argent des caisses du club. Après un long épisode judiciaire et de nombreux recours de la défense, en 1980, il est finalement condamné à 18 mois de prison avec sursis et doit payer 400000 francs de dommages et intérêts à son ancien club. 
des recherches restent à entreprendre sur ce point) qu'il a pu créer des liens avec d'autres présidents de clubs français ou à l'étranger. D'ailleurs, le Groupement des clubs autorisés (GCA), organisme qui représente les intérêts des clubs français professionnels, se montre rapidement favorable au projet du président de l'OM ${ }^{63}$. C'est donc une idée novatrice et portée par un acteur capable de mener à bien le projet qui est transmise aux dirigeants de l'UEFA dans le courant du printemps 1967.

\section{III - UN PROJET NEUTRALISÉ}

C'est précisément en date du 19 mai que Marcel Leclerc adresse son projet au président de l'UEFA, Gustav Wiederkehr. À noter qu'il essaie d'utiliser toutes les ressources symboliques à sa disposition pour soutenir son idée, puisqu'il emploie le papier à en-tête officiel de son journal, Téle-Magażine, et signe son courrier en tant que président de l'Olympique de Marseille ${ }^{64}$.

\section{a) Convaincre les instances du football international}

Marcel Leclerc cherche des appuis auprès des instances footballistiques internationales en vue de réaliser son projet et, parallèlement à sa démarche auprès de l'UEFA, envoie également une lettre au président de la FIFA, sir Stanley Rous ${ }^{65}$. Contacter Rous a l'avantage de contourner l'instance européenne ainsi que de commencer une discussion avec un autre organisateur d'épreuves, à savoir le président de la Coupe des villes foires. En outre, en raison des tensions occasionnelles qui existent entre l'UEFA et la FIFA ${ }^{66}$, Marcel Leclerc pense peut-être que le dirigeant anglais pourrait être un soutien de poids, et ce d'autant plus que de vives discussions existent alors sur le sujet du transfert de la Coupe des foires entre les mains de l'UEFA. Cependant, Stanley Rous va se montrer loyal vis-à-vis de l'Union.

En effet, il indique tout d'abord à Leclerc que c'est avec elle qu'il doit traiter de la question. Puis, et parallèlement à sa réponse au dirigeant marseillais, l'Anglais contacte le secrétaire général de l'UEFA afin de l'informer de

\footnotetext{
${ }^{63}$ Lettre de Marcel Leclerc à Gustav Wiederkehr, 29 mai 1967. UEFA, cote RM0002713 (Correspondence related to UEFA endorsed competitions), pochette : Coupe télémagazine.

${ }^{64}$ Ibid.

${ }^{65}$ Nous n'avons pas retrouvé cette lettre. En revanche, dans un courrier du 23 mai, il est indiqué que Marcel Leclerc a contacté Stanley Rous en date du 19 mai.

${ }^{66}$ Sur les tensions entre l'UEFA et la FIFA à cette époque voir : TOMLInson, Alan ; Sudgen, John. 1997. "Global power struggles in world football: FIFA and UEFA, 1954-1974, and their legacy », International Journal of the History of Sport, $\mathrm{n}^{\circ} 14$, automne, p. 1-25.
} 
l'affaire, et lui transmet même une copie du projet ${ }^{67}$. En dirigeant expérimenté du football - il a été secrétaire général de la Football Association de 1934 à 1961, puis vice-président de l'UEFA de 1959 à 1961, et enfin président de la FIFA depuis 1961 -, il sait tous les risques que comportent des projets développés par des acteurs privés pour la légitimité et le pouvoir des organes footballistiques supranationaux. En ce sens, il est absolument nécessaire d'être solidaire face à ce type d'initiative.

Comment l'instance européenne réagit-elle face au projet du président de l'OM ? Il semble qu'à cette période, malgré le ton enthousiaste du courrier de Marcel Leclerc, son idée de compétition n'est pas forcément accueillie avec le même sentiment par les dirigeants de l'UEFA. En effet, dans sa réponse de remerciements à Stanley Rous, Hans Bangerter indique :

« Je ne pense pas que cette compétition a la moindre chance d'être acceptée par l'UEFA. Premièrement, comme vous l'avez justement dit dans votre lettre à $\mathrm{M}$. Leclerc, il y a déjà assez de compétitions de clubs qui existent. Deuxièmement, je sais que les clubs latins ne sont pas intéressés à une autre compétition de cet ordre ${ }^{68}$.»

Quelques jours après avoir reçu la réponse de Stanley Rous, et restant sans nouvelles de l'UEFA, Marcel Leclerc écrit à nouveau à son président. Il n’hésite pas à souligner que l'initiative « semble intéresser vivement la plupart des grandes villes méditerranéennes ${ }^{69}{ }$ et rappelle qu'il a également obtenu le support du GCA. Fort de ses soutiens, il a même pris les devants et indique qu'une réunion en vue de discuter de l'épreuve entre lui et des représentants des clubs concernés est déjà programmée dans le courant du mois de juin à Paris $^{70}$. Cependant, il est désormais conscient, après l'échange avec Stanley Rous, qu'il doit absolument obtenir l'approbation de l'UEFA pour organiser l'épreuve et espère ainsi que l'Union sera « désireuse d'aider à la promotion de tout ce qui concerne l'heureux développement du football en Europe, se penchera avec intérêt sur cette initiative ${ }^{71} »$. C'est pourquoi Gustav Wiederkehr et Hans Bangerter sont invités à l'assemblée qui se déroulera dans la capitale

\footnotetext{
${ }^{67}$ Lettre de Stanley Rous à Hans Bangerter, 23 mai 1967. UEFA, cote RM0002713 (Correspondence related to UEFA endorsed competitions), pochette : Coupe télémagazine.

${ }^{68}$ Lettre de Hans Bangerter à Stanley Rous, 29 mai 1967. UEFA, cote RM0002713 (Correspondence related to UEFA endorsed competitions), pochette : Coupe Télémagazine. Courrier traduit de l'anglais par nos soins.

${ }^{69}$ Lettre de Marcel Leclerc à Gustav Wiederkehr, 29 mai 1967. UEFA, cote RM0002713 (Correspondence related to UEFA endorsed competitions), pochette : Coupe Télémagazine.

${ }^{70}$ Ibid.

${ }^{71}$ Ibid.
} 
française. S'il ne s'agit pas pour le dirigeant marseillais de s'affranchir totalement du pouvoir des instances dirigeantes, ce courrier indique néanmoins que son entreprise est déjà bien avancée. De fait, Leclerc met quasiment l'UEFA devant le fait accompli. Cette manière téméraire de procéder ne bénéficie sans doute pas à son initiative, les dirigeants de l'Union s'estimant, comme indiqué plus haut, les garants de l'administration des compétitions européennes. À ce titre, ils souhaitent être les premiers informés de ce type de démarche. Révélatrice de cette posture, la réponse faite par le secrétaire général de l'UEFA à Marcel Leclerc, en date du 7 juin, est courte. Bangerter lui indique simplement que l'entité européenne n'autorise pas l'organisation de l'épreuve, la raison principale étant que le nombre de matchs qui se disputent sur le continent est déjà trop conséquent ${ }^{72}$. C'est donc une fin de non-recevoir que se voit transmettre le président de l'Olympique de Marseille.

Malgré cette réponse négative, Marcel Leclerc ne se montre pas abattu et persiste dans son entreprise. L'obstination dont il fait preuve est d'ailleurs l'une de ses forces, et il faut souligner qu'il est régulièrement décrit comme un «lutteur» ou un « battant» par les journalistes ${ }^{73}$. Quelques jours plus tard, il répond à l'UEFA et indique qu'il espère « qu'avec un peu de temps, avec l'accord des clubs et des fédérations intéressées [que l'UEFA] après nouvel examen, pourra, en meilleure connaissance de cause, réviser son premier jugement $^{74} \gg$. En dépit du manque de soutien de l'instance européenne, Marcel Leclerc poursuit donc son projet et continue à prospecter en vue de sa réalisation.

Il tient notamment Stanley Rous au courant des événements et l'invite aux réunions prévues ${ }^{75}$. Si celle envisagée le 12 juin parait finalement avoir été abandonnée, une autre assemblée est fixée au 26 du même mois ${ }^{76}$. À ce stade de nos recherches, il est difficile de savoir si l'événement est organisé par ce jour-là et qui y prend part. Par contre, une chose est sûre : Marcel Leclerc continue à s'activer en vue de créer la compétition et décide de se rendre à

\footnotetext{
${ }^{72}$ Lettre de Hans Bangerter à Marcel Leclerc, 7 juin 1967. UEFA, cote RM0002713 (Correspondence related to UEFA endorsed competitions), pochette : Coupe Télémagazine.

${ }^{73}$ Comme l'atteste plusieurs articles du journal Le Monde qui traite des démêlés du président de l'OM avec la justice et avec son ancien club à la fin des années 1970. En outre, les éditoriaux écrits chaque semaine par Marcel Leclerc en ouverture des numéros de Télé-Magazine sont souvent offensifs et il n'est pas rare que le propos critique ouvertement les instances du football français et international.

${ }^{74}$ Lettre de Marcel Leclerc à Hans Bangerter, 13 juin 1967. UEFA, cote RM0002713 (Correspondence related to UEFA endorsed competitions), pochette : Coupe Télémagazine.

${ }^{75}$ Lettre de Marcel Leclerc à Stanley Rous, 21 juillet 1967. UEFA, cote RM0002713 (Correspondence related to UEFA endorsed competitions), pochette : Coupe Télémagazine.

${ }^{76}$ «Le championnat latin que mijote l'OM », France football, 13 juin 1967.
} 
Genève lors de la réunion préparatoire des compétitions européennes de la saison 1967-1968 qui doit se dérouler le 5 juillet 1967. La séance réunit les délégués des clubs qui sont qualifiés pour les épreuves continentales de l'UEFA (soit les champions de chaque pays ainsi que les vainqueurs des coupes nationales, accompagnées des deux derniers vainqueurs de la saison précédente), ce qui n'est pas le cas de l'Olympique de Marseille. Dans la cité de Calvin, Marcel Leclerc espère pouvoir s'entretenir directement avec Gustav Wiederkehr et Hans Bangerter. Mais, étant informé de la présence de nombreux dirigeants de clubs ainsi que de journalistes de plusieurs pays européens, nous pouvons supposer que son objectif est également de continuer à exposer son idée et d'obtenir de nouveaux appuis en vue de sa concrétisation.

\section{b) D’une épreuve européenne à une compétition régionale}

À Genève, Leclerc réussit à discuter avec le président et le secrétaire général de l'UEFA. En effet, le lendemain de la réunion tenue sur les bords du Lac Léman, le président de l'OM se permet d'écrire à Gustav Wiederkehr qu'il a bien retenu les demandes de l'instance européenne concernant l'organisation de l'épreuve ${ }^{77}$. Selon lui, celles-ci se résument comme suit:

- D'avoir l'accord des fédérations nationales qui ont un club qui participe à l'épreuve.

- De convoquer des représentants de ces fédérations dans le comité d'organisation du tournoi.

- Que l'UEFA dispose d'un droit de contrôle sur le déroulement de la compétition.

Continuant sur sa lancée, le dirigeant marseillais reprend rapidement le règlement du futur tournoi. Dans cette nouvelle version, la place conférée aux instances footballistiques est confirmée, puisque le comité d'organisation comptera un représentant de la FIFA, un de l'UEFA et un de chaque fédération nationale concernée, qui s'ajouteront aux représentants des clubs participants $^{78}$. Ayant entendu les requêtes de l'élite dirigeante de l'UEFA, Marcel Leclerc peut donc s'attendre à un changement de posture de l'Union vis-à-vis de son projet.

\footnotetext{
${ }^{77}$ Lettre de Marcel Leclerc à Gustav Wiederkehr, 6 juillet 1967. UEFA, cote RM0002713 (Correspondence related to UEFA endorsed competitions), pochette : Coupe Télémagazine.

${ }^{78}$ « Règlement sportif et financier de la Coupe Télé-Magazine (saison 1967-1968) - sur invitation », LECLERC, Marcel, non daté. UEFA, cote RM0002713 (Correspondence related to UEFA endorsed competitions), pochette : Coupe Télémagazine.
} 
Effectivement, quelques jours plus tard, par la voix de son président, l'UEFA confirme qu'elle pense pouvoir donner son accord à l'organisation du futur tournoi mais, avant de définitivement trancher sur la question, attend encore l'accord des fédérations nationales concernées ${ }^{79}$. Comment expliquer ce revirement ? Outre d'avoir été écoutée et conviée au sein du comité d'organisation, si l'UEFA autorise finalement le projet, c'est sans doute parce que celui-ci est nettement moins ambitieux que ne pouvait le laisser présager la première version du projet et, dès lors, ne concurrence plus vraiment ses épreuves. Marcel Leclerc lui-même semble conscient de cette situation puisqu'il écrit à Gustav Wiederkehr que la compétition méditerranéenne constituera « un peu le pendant de [la Coupe intertoto] en ce qui concerne les pays du centre et du nord de l'Europe de l'Ouest ${ }^{80} »$. Le futur tournoi n'est donc pas à considérer comme épreuve européenne, mais plutôt comme une épreuve régionale, dans la lignée de la Mitropa cup ou de la Coupe des Balkans.

Le projet de compétition est en phase d'être accepté par l'UEFA mais un point majeur reste à régler : celui de la participation des équipes. Selon le nouveau règlement, il est envisagé que la première édition de l'épreuve convoquera uniquement des clubs espagnols tels Barcelone, Saragosse, l'Atletico de Madrid, et français comme les Girondins de Bordeaux, l'Olympique de Marseille et le FC Nantes. Ces formations correspondent aux souhaits initiaux de Marcel Leclerc, à savoir d'inviter des formations de pointe, celles-ci ayant toutes terminé dans le haut du classement de leur championnat respectif. À la fin juillet, la Fédération française donne son accord pour la participation de ses clubs ${ }^{81}$. Toutefois, la réponse de son homologue espagnol se fait attendre. En fait, cette association a toujours été très loyale envers l'UEFA et s'est rapidement montrée proactive en vue de son développement, position qui témoigne de l'investissement progressif du franquisme dans ce que l'historien Juan Antonio Simon a appelé la « diplomatie de la balle ${ }^{82}$ ». Déjà, à la fin des années 1950, elle avait refusé de participer aux joutes développées par le Comité interliaison des ligues professionnelles, indiquant que celles-ci

\footnotetext{
${ }^{79}$ Lettre de Gustav Wiederkehr à Marcel Leclerc, 17 juillet 1967. UEFA, cote RM0002713 (Correspondence related to UEFA endorsed competitions), pochette : Coupe Télémagazine.

${ }^{80}$ Lettre de Marcel Leclerc à Gustav Wiederkehr, 6 juillet 1967. UEFA, cote RM0002713 (Correspondence related to UEFA endorsed competitions), pochette : Coupe Télémagazine.

${ }^{81}$ «Bureau fédéral. Séance du samedi 8 juillet 1967 à Rouen (Chambre de commerce) », Delaunay, Pierre, non daté. Archives de la Fédération française de football (FFF), cote : Livre du bureau fédéral.

${ }^{82}$ Simon, Juan Antonio. 2012. «La diplomacia del Balón. Deporte y relaciones internacionales durante el franquismo », História e Cultura, n 4, décembre, p. 165-189.
} 
devraient être organisées sous l'égide de l'UEFA ${ }^{83}$. Ici encore, elle va jouer un rôle en vue de renforcer l'autorité de l'Union, puisqu'en septembre la Fédération espagnole indique au secrétaire Hans Bangerter qu'elle n'a pas donné suite à la proposition de Marcel Leclerc. Le nombre important d'épreuves déjà existantes et le fait que, sauf Barcelone, «aucun autre club ne s'est montré intéressé à la question ${ }^{84}$ » sont les principaux arguments évoqués par son secrétaire général.

En raison de ce manque de soutien, le projet du dirigeant marseillais est finalement abandonné. Douze ans après, Marcel Leclerc n’a donc pas réussi la même entreprise que ses confrères de L'Équipe, à savoir de forcer les instances européennes à reprendre leur projet et de lancer une épreuve continentale. Si la position de Leclerc et ses ambitions se rapprochent, en partie, des journalistes du quotidien parisien, l'espace footballistique européen est entré dans une nouvelle phase et, surtout, il est désormais dominé par une organisation qui a progressivement affirmé sa position au sein de celui-ci : l'UEFA.

\section{CONCLUSION}

\section{ÉTUDIER LE LONG CHEMIN VERS LA LIGUE DES CHAMPIONS}

Le projet porté par Marcel Leclerc révèle que la deuxième partie des années 1960 est un moment clé pour l'histoire des épreuves européennes de football. En effet, l'argumentaire développé dans le projet du dirigeant marseillais reprend des griefs qui sont portés par plusieurs acteurs du football européen (dirigeants de clubs et journalistes) envers le déroulement des épreuves européennes. Cependant, son échec amène à deux constats. D’une part, il souligne la position centrale occupée par l'UEFA en matière d'organisation des épreuves européennes. Dorénavant, sans son accord, aucune modification ou réforme ne semble possible sur le sujet. D'autre part, l'échec du projet de Leclerc indique également que le nombre de compétitions européennes est désormais bien établi et qu'il est de plus en plus difficile, dans ce calendrier chargé, de créer de nouvelles épreuves supranationales.

\footnotetext{
${ }^{83}$ Lettre de la Fédération espagnole au comité de la Coupe internationale des villes de foires, 19 janvier 1959. UEFA, cote RM0000749 (ExCo meetings, 1954-1959), pochette : Comité exécutif de l'UEFA. Séance du 11Xbre 59 à Paris.

${ }^{84}$ Lettre de la Fédération espagnole à l'UEFA, 25 septembre 1967. UEFA, cote RM0002713 (Correspondence related to UEFA endorsed competitions), pochette : Coupe Télémagazine.
} 
Dans les deux décennies qui suivent, l'emprise de l'UEFA sur le football européen va se renforcer, en particulier avec la création de la Coupe UEFA qui remplace la Coupe des villes de foires. Le nom donné à l'épreuve est d'ailleurs hautement symbolique de cette assise de l'Union sur l'organisation des compétitions européennes, puisqu'elle n'hésite pas à apposer, et imposer, son propre nom à une épreuve qui existait déjà. L'UEFA va néanmoins répondre à certaines critiques qui sont régulièrement émises par les dirigeants de clubs, ou les journalistes, à l'égard de ses compétitions. Ainsi, une synchronisation des calendriers s'opère progressivement avec la mise en place de véritables "semaines européennes », dont une bonne partie des matchs sont désormais retransmis en direct sur le petit écran.

Matchs de poule, forte présence de la télévision, volonté d'accroittre les recettes des clubs. Indéniablement, le projet porté par Marcel Leclerc comporte déjà des ingrédients de la Ligue des champions qui voit le jour au tournant des années $1990^{85}$. En ce sens, celui-ci peut être considéré comme une Ligue des champions avant l'heure. Si elle ne se concrétise pas, l'idée du président de l'OM témoigne cependant de la commercialisation et de la médiatisation croissantes que connait le football européen. Il s'agit dès lors, dans de futures recherches, d'étudier plus dans le détail la période qui va des années 1970 aux années $1980^{86}$, démarche qui permettra de véritablement saisir les enjeux (économiques, institutionnels, sportifs, voire politiques) de la création de la Ligue des champions.

\footnotetext{
${ }^{85}$ Sur les nouveautés apportées par la Ligue des champions voir : Holt, Matthew. 2007. «Global Success in Sport: The Effective Marketing and Branding of the UEFA Champions League », International Journal of Sport Marketing \& Sponsorship, n 9, hiver, p. 51-61.

${ }^{86}$ Quelques éléments provisoires, en particulier sur les dirigeants de l'UEFA, ont été mis en lumière par Manuel Schotté : Sснотте́, Manuel. 2014. «La structuration du football professionnel européen. Les fondements sociaux de la prévalence de la "spécificité sportive" ", Revue française de socio-économie, $\mathrm{n}^{\circ}$ 13, numéro spécial, printemps, p. 99-120.
} 